\title{
Migrants' Experiences in the Healthcare System
}

\author{
Marie-Louise Luiking \\ Leiden University, The Netherlands \\ marie-louise@luiking.com \\ Harshida Patel \\ University of Gothenburg, Sweden \\ harshida.patel@gu.se
}

\begin{abstract}
Mass migration in Europe raises many issues of societal concern. Migrants struggle with complex health problems related to causes and consequences of migration. It is no longer a question about migrants having unique health needs, concerns and challenges. The effects of language and cultural differences, lack of access to transportation and other barriers are common. These barriers hamper nurses and other healthcare professionals to deliver high quality care. An essential dimension of quality of care mirroring both patients' expectations and their exposure is the migrant patient's experience of healthcare. This chapter addresses the factors associated with migrants' healthcare experiences in the host country. A model presented here may increase nursing professionals'awareness and help to provide person centred care and overcome cultural differences.
\end{abstract}

\section{Migration}

The picture of migration, is vastly more complex than at first glance and ease of movement promises greater complexity in the future. The International Organisation for Migration (see http://www.iom.int/key-migration-terms) defines a migrant as: 'any person who is moving or has moved across an international border or within a state away from his/her habitual place of residence, regardless of the person's legal status, voluntary or involuntary, causes, and the length of the stay.' With the number of international migrants escalating (United Nations, 2016), it becomes more acute that migrants face numerous barriers to access an appropriate level of healthcare (World Health Organisation, 2008).

\section{Addressing Migrant Health Issues}

The media conversation often sticks narrowly to the question of how the migrant arrives. The focus on such migration issues sidetracks the important needs of migrant populations, by moving the focus away from addressing 
health needs of persons in the host countries. It also stalls needed adjustments of the rigid healthcare systems that are largely unequipped to cater for migrants in need of care.

The health issues faced by migrant populations are similar to those faced by the general population but are often compounded by their migratory history. Mobility results in poor continuity of care and simultaneously increases the need for care (Kugel \& Zuroweste, 2010). Poor mental and physical health lead to a greater risk of morbidity and mortality in comparison to the rest of the population of the host country.

\section{Access to Healthcare}

Access has been found to be a leading health indicator (Anderson, Scrimshaw, Fullilove, Fielding, \& Normand, 2003). Health is one of the most fundamental resources for an individual's potential for development to be fulfilled (Marmot, Friel, Bell, Houweling \& Taylor 2008), as well as a key component for an immigrant's integration into the host society and the labour market. However, findings from research show a decline in health status among the refugees after settling in their host country (Newbold, 2005; Salman \& Resick, 2015; Taylor et al., 2014).

Migrants struggle with similar challenges as other underserved populations regarding access to healthcare, but face the additional barriers of mobility, language, cultural differences, and lack of familiarity with local healthcare services. Migrants on the move are brought out of their provider networks, reducing further access. It is even more complicated for the undocumented migrants to contact healthcare as they often fear for deportation.

An extensive variety of barriers is reported in literature including: healthcare professionals' views of migrants' health issues, problems with adaptation and little understanding of migrants' expectations of healthcare services within the host country. (Hakonsen, Lees, \& Toverud, 2014; Kalich, Heinemann, \& Ghahari, 2016; Suphanchaimat, Kantamaturapoj, Putthasri, \& Prakongsai, 2015). These barriers hamper nurses and other healthcare professionals to deliver high quality care. An essential dimension of quality of care mirroring both patients' expectations and their exposure is the migrant patient's experience of healthcare.

The main challenges for healthcare professionals are lack of awareness of the migrants' specific healthcare problems, language and intercultural communication problems, as well as access and integration of migrant seekers into the healthcare system. Awareness of these conditions is mandatory to ensure good clinical practice for this patient population, which has a huge 
burden in chronic, infectious, mental, and neurological diseases (Salman \& Resick, 2015).

The major impact factors on migrants' health are linked to experiences and exposure in the country of origin, in camps and en route and in the process of immigration into the host country and living in foreign countries. Migrants' health is also affected by psychological problems and by infectious diseases due to the exceptional and hazardous conditions of their journey.

High quality patient care should be effective, efficient, accessible, patient centred, equitable and safe (WHO, 2008). Due to the extensive barriers, however, migrant healthcare is not always meeting high quality care standards. Of all healthcare professionals, nurses are the largest staff group which also has most direct patient contact. Nurses are therefore a staff group that is particularly important in ensuring the quality of migrant patient care.

\section{Healthcare-Seeking Behaviour: Influencing Factors}

Healthcare seeking behaviour (HCSB) is a term that refers to patterns of utilization, treatment seeking, delay in seeking care, predicting the future probable burden of diseases, facilitation of the health status, early diagnosis, complete and effective treatment, and complication control. HCSB relies on time and context and are associated with social, cultural, economic, disease pattern and issues related to health services (Poortaghi et al., 2015). Multiple factors such as educational level (Ahmed et al., 2016; Chau et al., 2018; Dawood et al., 2017, Jurcev-Savicevic \& Kardum, 2012), occupation, marital status, economic status, age and sex, healthcare costs, type and severity of illness, distance and physical access, and perceived quality of service provision (Caitlin \& Austen, 2009; Tipping \& Segall, 1995) are associated with HCSB. Further factors include time, insecurity, social status, language barriers, lack of information (Ahmed et al., 2016; Kalich et al., 2016) and cultural differences (Ahmed et al., 2016; Chau et al., 2018; Kalich et al., 2016).

The migrant population is diverse and each person has individual health needs (Luiking et al., 2018). Indeed, the most recent project conducted by our research group, a meta ethnography study (Luiking et al., 2018) showed that a multitude of factors influence the HCSB decision-making process. Migrants seek care according to their needs and legal status and they often consult trusted individuals within their own community about how to address a health problem before seeking formal help (Luiking et al., 2018). Further considerations are time and financial resources (Herdman et al., 2016; Lattof, 2018). The framework derived from our investigation provides a more comprehensive overview on HCSB in migrants (Luiking et al., 2018) and de- 
scribes the personal and healthcare system-inherent barriers and enablers to accessing and provision in this population. These include personal factors, the healthcare system, accessing healthcare, the encounter and healthcare experiences (see Figure 1 on p. 102).

The decision to seek care is therefore not a simple move taken by an individual person, instead, it is a more complex process that is embedded within a social, cultural and community context. Importantly, modern Information Technology may play a crucial part in health seeking in the migrant population.

\section{Determinants of Health Seeking Behaviours}

Self-efficacy and attitude may also play an important role in HCSB. Selfefficacy is the belief in one's competence to tackle tasks and to cope with adversity in a broad range of stressful or challenging situations (Bandura, 1977). For example, while ill and in need of healthcare, self-efficacy emerged as a dominant determinant of engagement in health compromising or health promoting behaviours (Bandura, 1977, 1993). However, the relationship between self-efficacy and healthcare seeking behaviours within the migrant population are to date unknown. Studies on the health of migrants in Europe focus on care experiences of those actively seeking care. Little is known about factors that prevent migrants from seeking care and self-efficacy may play an important role in ensuring personal health. The role and importance of self-efficacy, personal networks and indeed, modern technologies such as internet or apps on mobile phones in seeking care has not been reported, despite technology becoming increasingly important in decision-making and information seeking in all areas of life. An investigation of the role of selfefficacy in HCSB and associated resources such as personal networks and information technology will provide new perspectives on how to promote effective care-seeking in the migrant population.

\section{Literature Review of Migrant Patients' Experiences of Healthcare}

Insight into health disparities and associated barriers to care in the group of migrant patients can be obtained by looking into the migrant patients' perspective of their healthcare (Tilburt, 2010). Although a fair amount of attention in research has been given to migrant health, there was no systematic synthesis of the evidence on migrant patient experience.

To address this gap in knowledge a review was done to synthesize published literature related to adult migrant patients' experiences of healthcare and their healthcare seeking behaviours. The objective was a comprehen- 
sive overview of barriers at various stages of the patient journey. Metaethnography was chosen to synthesize the studies; this enables the interpretation of the findings from the individual studies into a larger, whole picture that provides deeper understanding of an issue (Noblit \& Hare, 1988). The population included in this review were adult migrant patients, but not specific maternity care.

\section{The Review Process}

Any peer-reviewed, qualitative research study in English, exploring migrant patients' experiences of healthcare published between January 2000-June 2016 was considered. The search in 7 databases initially delivered 264 studies, which were further assessed according to the inclusion criteria and for their study quality. 27 studies were included in the meta ethnographic analysis.

Hundred sixteen first and second order constructs were identified across the studies, which were condensed and interpreted into 15 third order constructs. These third order constructs were contextualised into the following dimensions: (1) personal factors; (2) healthcare system; (3) access to healthcare; (4) the encounter; and (5) healthcare experience (Luiking et al., 2018).

\section{Findings}

The third order constructs, dimensions are described below. In italics are quotations from the studies in the review to illustrate the dimensions.

\section{Personal Factors}

This dimension comprises a number of constructs that define a migrant patient's personality and inform about their health-seeking behaviours, such as a person's enculturation or society of origin with its religious or value system, but also life experiences. In some cases, exceptionally traumatic experiences such as becoming a refugee because of war and being forced to migrate are powerful: 'I did not want to live because of what is gone. I was eating, sleeping only [...] I tried to forget it but I can't. My history, my life is stamped into my heart.' (Fang, Sixsmith, Lawthom, Mountian, \& Shahrin, 2015)

In effect these personal factors determine a person's overall life context (Biswas, Kristiansen, Krasnik, \& Norredam, 2011; Krupic, Sadic, \& Fatahi, 2016; Seffo, Krupic, Grbic, \& Fatahi, 2014).

\section{Healthcare System}

The healthcare system and legal framework of the host country determines the care provision available for natives as well as for migrant patients. De- 
pending on the healthcare model, service provision and the quality of care might differ between a migrant's home country and host country. This can result in a mismatch between the care provided and the individual's expectations from the healthcare system: 'It is not easy for a foreigner to cure our diseases. The Chinese [...] when they go to school, they don't teach them about African sicknesses. [...]' (Lin et al., 2015)

\section{Access to Healthcare}

This dimension addressed the barriers or enablers to healthcare in the host country. When a need for services is identified by migrants, their socioeconomic and legal status can affect their access to services. To even access the correct service, the individual migrant and/or their families need to know how to go about such access. Language difficulties and lack of information can serve to adversely affect their rights. An additional issue is that service providers can turn out to be gatekeepers to the required services (Biswas et al., 2011; Legido-Quigley, Nolte, Green, la Parra, \& McKee, 2012; Lin et al., 2015). Care-seeking can become outright dangerous for undocumented persons: 'If I go to the doctor and the doctor is a very good Danish person, a good citizen, then maybe he will call the police. And then I would be handed over to the police and then I would have a great problem. Then my life is risky.' (Biswas et al., 2011). These issues can have a detrimental effect on the individual migrant's health and they may apply alternative health-seeking strategies (Biswas et al., 2011; Krupic et al., 2016; Main, 2016) 'When I am not feeling well, I will call my doctor in my home country. He will ask about my symptoms and tell me what medicine to get over the phone' (Lin et al., 2015).

\section{The Encounter}

When individual migrants do access the services, the way they are treated as a person and as a patient is determined by the staff they encounter. This juncture has huge implications for the trajectory of a person's care. Yet the encounter can prove problematic due to language difficulties and a lack of mutual knowledge of how to act in a culturally appropriately way. This also covers the misunderstandings of what an individual migrant wants from service providers, what they are used to in their own countries and what the service provider states that the person with migrant status needs 'In Poland, people go to the doctor more often. Pregnancy is the best example. In Poland, your pregnancy is over-medicalized. You are constantly under medical surveillance, constantly tested. Here it is the opposite [...] pregnancy is not an illness; you don't need to be on sick leave all these months [...]' (Main, 2016) 
It is therefore important for service providers to treat the individual in an holistic manner, ascertaining their expectations and perceived need. Such care is at the heart of person-centeredness. Within a true person-centred approach, the individual's cultural background is fundamental to effective care.

\section{The Healthcare Experiences}

This dimension comprises the constructs of healthcare needs as being 'met' or 'unmet.' Healthcare needs need to be satisfied in terms of emotional support or information. The overall positive or negative evaluation of a healthcare experience depends on whether a migrant patient feels their care and information needs and expectations have been met or not. Migrant patients use the familiar healthcare system of their home country as a template to compare and evaluate the care they received in the host country. 'I wouldn't go to the [British] NHS. My experience of medical care is good [in Spain] [...] The biggest difference about Spain and England is that people care about nursing and the people in England they are more worried about targets.' (Legido-Quigley et al., 2012)

From the findings, a model has been designed using the five dimensions and grounded in a person-centred care approach (Figure 1). This model is in the form of a flow diagram that illustrates the antecedents and succedents of the migrants' healthcare experience. These include lack of linguistic abilities, clashing cultures or social or cultural taboos that may inhibit them from seeking healthcare and, when found, of making the best use of it. This model may help healthcare providers to identify and address antecedents to poor quality migrant healthcare, identify weak points, improve the organisation and help healthcare professionals to provide person-centred care to migrant patients.

The model points out that language difficulties and differences with migrants' experience in the home countries are important factors in all stages. The comparison with migrants' experience in the home countries implies that the mere provision of linguistically congruent information might not serve all migrants' needs, as it is generally aimed at people who already understand the workings of a healthcare system. However, migrant patients may lack this basic understanding. Information should therefore be adapted to the different needs of 'migrants' (for example refugees and illegal immigrants). This would include providing a basic explanation of access to and routes through the healthcare system. In addition, understanding of the host cultures, how patients relate to healthcare professionals and which behaviours are acceptable, or not, should be explained. 


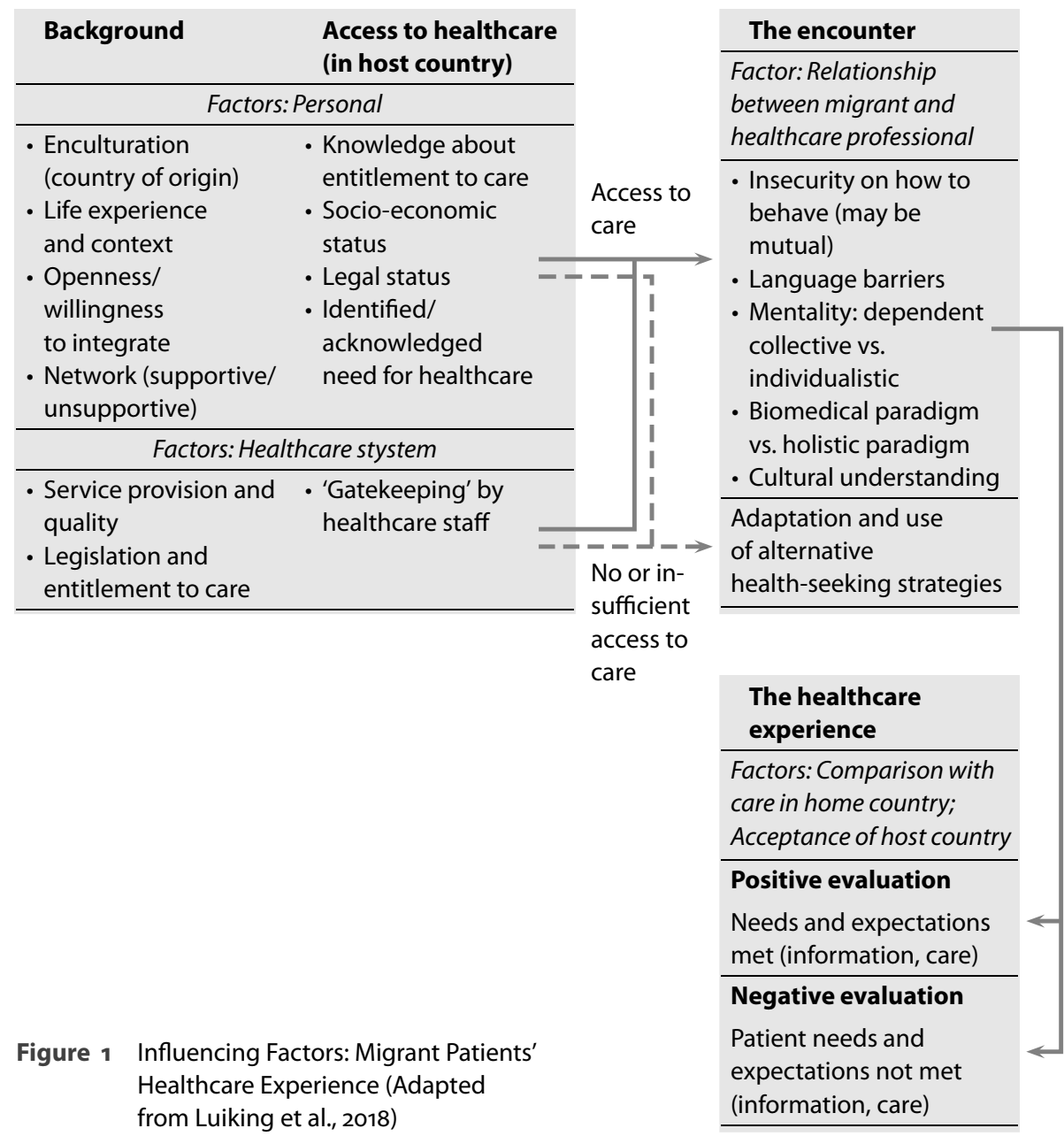

\section{Clinical Relevance}

For the nurse in her encounter with a migrant the flow diagram shows the personal factors and healthcare system factors that ante cede and shape the encounter. It also shows the factors of the encounter itself which matter and the factors which determine how the migrant experiences the encounter subsequently. Healthcare professionals, nurses often perceive migrants as 'others', as being different from 'them.' This 'othering' prevents an open-minded encounter and healthcare professionals, nurses need to be mindful of potential prejudice. Migrants, like every individual, are shaped by 
their culture of origin as well as their life experience and they have thus diverse needs. Migrants' backgrounds may be alien to healthcare professionals and difficult to understand. Awareness of one's own preconceived ideas can enable healthcare professionals to approach migrants as the unique individuals they are: people with specific health needs that need special attention, just as any other patient (Grove \& Zwi, 2006).

The model can help the healthcare professional, nurse as a reminder of what determines the healthcare experience of a migrant. Perhaps with the model in hand it will be easier for the healthcare professional, nurse to adapt the care provided to fit the migrant patient better.

\section{References}

Ahmed, S., Shommu, N. S., Rumana, N., Barron, G. R., Wicklum, S., \& Turin, T. C. (2016). Barriers to access of primary healthcare by immigrant populations in Canada: A literature review. Journal of Immigrant and Minority Health, 18(6), 1522-1540.

Anderson, L. M., Scrimshaw, S. C., Fullilove, M. T., Fielding, J. E., Normand, J. (2003). Culturally competent healthcare systems: A Systematic review. American Journal of Preventive Medicine, 24(3), 68-79.

Bandura, A. (1977). Self-efficacy: Toward a unifying theory of behavioral change. Psychol Review 84(2), 191-215.

Bandura, A. (1993), Perceived self-efficacy in cognitive development and functioning. Educational Psychologist, 28(2), 117-148.

Biswas, D., Kristiansen, M., Krasnik, A., \& Norredam, M. (2011). Access to healthcare and alternative health-seeking strategies among undocumented migrants in Denmark. British Medical Council Public Health, 11(560). Retrieved from http://doi.org/10.1186/1471-2458-11-560

Caitlin, M., \& Austen, D. (2009). Health care seeking behaviour in Somalia: A literature review (Report 10). Retrieved from https://www.unicef.org/somalia/ SOM_HealthcareseekingbehaviourReport_10-WEB.pdf

Chau, P. H., Moe, G., Lee, S. Y., Woo, J., Leung, A. Y. M., Chow, C. M., Kong, C., Lo, W. T., Yuen, M. H., \& Zerwic, J. (2018). Low level of knowledge of heart attack symptoms and inappropriate anticipated treatment-seeking behaviour among older Chinese: A cross-sectional survey. Journal of Epidemiology and Community Health, 72(7), 645-652.

Dawood, O. T., Hassali, M. A., Saleem, F., Ibrahim, I. R., Abdulameer, A. H., \& Jasim, H. H. (2017). Assessment of health seeking behaviour and self-medication among general public in the state of Penang, Malaysia. Pharmacy Practice, 15(3), 991.

Fang, M., Sixsmith, J., Lawthom, R., Mountian, I., \& Shahrin, A. (2015). Experiencing 'pathologized presence and normalized absence:' Understanding health related experiences and access to healthcare among Iraqi and So- 
mali asylum seekers, refugees and persons without legal status. British Medical Council Public Health, 15(1), 923.

Grove, N., \& Zwi, A.(2006). Our health and theirs: Forced migration, othering, and public health. Social Science \& Medicine, 62(8), 1931-1942.

Herdman, M. T., Maude, R. J., Chowdhury, M. S., Kingston, H. W., Jeeyapant, A., Samad, R., Karim, R., Dondorp, A. M., \& Hossain, M. A. (2016). The relationship between poverty and healthcare seeking among patients hospitalized with acute febrile illnesses in Chittagong, Bangladesh. PLoS One, 11(4), e0152965.

Hakonsen, H., Lees, K., \& Toverud, E. L. (2014). Cultural barriers encountered by Norwegian community pharmacists in providing service to non-Western immigrant patients. International Journal of Clinical Pharmacy, 36(6), 11441151.

Jurcev-Savicevic, A., \& Kardum, G. (2012). Health-care seeking behaviour for tuberculosis symptoms in Croatia. The European Journal of Public Health, 22(4), 573-577.

Kalich, A., Heinemann, L., \& Ghahari, S. (2016). A scoping review of immigrant experience of health care access barriers in Canada. Journal of Immigrant and Minority Health, 18(3), 697-709.

Krupic, F., Sadic, S., \& Fatahi, N. (2016). Contact with health-care service expectation and reality of situation experienced by immigrants seeking Swedish health-care. Materia Socio-Medica, 28(2), 141-145.

Kugel, C., \& Zuroweste, E. (2010). The state of health care services for mobile poor populations: History, current status, and future challenges. Journal of Health Care for the Poor and Underserved, 21(2), 421-429.

Lattof, S. R. (2018). Health insurance and care-seeking behaviours of female migrants in Accra, Ghana. Health Policy and Planning, 33(4), 505-515.

Legido-Quigley, H., Nolte, E., Green, J., la Parra, D., \& McKee, M. (2012). The healthcare experiences of British pensioners migrating to Spain: A qualitative study. Health Policy, 105(1), 46-54.

Lin, L., Brown, K., Yu, F., Yang, J., Wang, J., Schrock, J., ... Wong, F. (2015). Healthcare experiences and perceived barriers to healthcare access: A qualitative study among African migrants in Guangzhou, Guangdong Province, China. Journal of Immigrant \& Minority Health, 17(5), 1509-1517.

Luiking, M., Heckemann, B., Ali, P., Dekker vanDoorn, C., Ghosh, S., Kydd, A., Watson, R., \& Patel, H. (2018). Migrants' healthcare experience: A metaethnography review of the literature. Journal of Nursing Scholarship. Retrieved from https://www.doi.org/10.1111/jnu.12442

Main, I. (2016). Biomedical practices from a patient perspective: Experiences of Polish female migrants in Barcelona, Berlin and London. Anthropology \& Medicine, 23(2), 1-17.

Marmot, M., Friel, S., Bell, R., Houweling, T. A., \& Taylor, S. (2008). Closing the gap 
in a generation: Health equity through action on the social determinants of health. The Lancet, 372(9650), 1661-1669.

Newbold, K. B. (2005). Self-rated health within the Canadian immigrant population: Risk and the healthy immigrant effect. Social Science \& Medicine, 60(6), 1359-1370.

Noblit, G., \& Hare, R. (1988). Meta-ethnography: Synthesizing qualitative studies (Vol. 11). London, England: Sage.

Poortaghi, S., Raiesifar, A., Bozorgzad, P., Golzari, S. E., Parvizy, S., \& Rafii, F. (2015). Evolutionary concept analysis of health seeking behavior in nursing: $A$ systematic review. BMC Health Services Research, 15(1), 523. Retrieved from https://doi.org/10.1186/s12913-015-1181-9

Salman, K. F., and Resick, L. K. (2015). The description of health among Iraqi refugee women in the United States. Journal of Immigrant and Minority Health, 17(4), 1199-205.

Seffo, N., Krupic, F., Grbic, K., \& Fatahi, N. (2014). From immigrant to patient: Experiences of Bosnian immigrants in the Swedish healthcare system. Materia Socio-Medica, 26(2), 85-89.

Suphanchaimat, R., Kantamaturapoj, K., Putthasri, W., \& Prakongsai, P. (2015). Challenges in the provision of healthcare services for migrants: A systematic review through providers' lens. BMC Health Services Research, 15(390). Retrieved from https://doi.org/10.1186/s12913-015-1065-z

Taylor, E. M., Yanni, E. A., Pezzi, C., Guterbock, M., Rothney, E., Harton, E., Montour, J., Elias, C., \& Burke, H. (2014). Physical and mental health status of Iraqi refugees resettled in the United States. Journal of Immigrant and Minority Health, 16(6), 1130-1137.

Tilburt, J. C. (2010). The role of worldviews in health disparities education. Journal of General Internal Medicine, 25(2), 178-181.

Tipping, G., \& Segall, M. (1995). Health care seeking behaviour in developing countries: An annotated bibliography and literature review. Brighton, England: Institute of Development Studies.

United Nations. (2016). International migration report 2015 (ST/ESA/SER.A/384). New York, NY: Author.

World Health Organisation. (2008). Overcoming migrants' barriers to health. Bulletin of the World Health Organisation, 86(8), 583-584.

S. Ličen, I. Karnjuš, \& M. Prosen (Eds.). (2019). Women, migrations and health: Ensuring transcultural healthcare (pp. 95-105).

Koper, Slovenia: University of Primorska Press.

https://doi.org/10.26493/978-961-7055-43-6.95-105 